\title{
Variations simultanées du poids vif et des quantités ingérées chez la jument
}

\author{
W. MARTIN-ROSSET, M. DOREAU *, R. ESPINASSE \\ avec la collaboration technique de H. DUBROEUCQ \\ I.N.R.A.-C.R.Z.V. Theix, 63122 Ceyrat \\ Unité élevage et alimentation du cheval \\ * Laboratoire de la lactation
}

\begin{abstract}
Résumé
Les variations à court terme du poids vif, consécutives à des variations provoquées des quantités ingérées, ont été mesurées chez 6 juments lourdes sèches alimentées avec un régime à base de foin de pré complémenté par 2 ou $4 \mathrm{~kg}$ de concentré. Le foin était distribué à 95,80 et 60 p. 100 des quantités ingérées à volonté selon un schéma en carré latin. Nous avons estimé que les variations du poids vif représentaient en grande partie celles du contenu digestif.

La variation du poids vif $(Y)$ est bien reliée à la variation de la quantité de matière sèche ingérée de fourrage $\left(\mathrm{X}_{1}\right)$ et d'aliment concentré $\left(\mathrm{X}_{2}\right)$. Lorsque les quantités de matière sèche ingérée de fourrage ou d'aliment concentré varient de $1 \mathrm{~kg}$, le poids vif de la jument varie respectivement de $3,48 \pm 0,40 \mathrm{~kg}$ ou de $3,45 \pm 0,95 \mathrm{~kg}$.
\end{abstract}

Mots clés: Cheval, jument, poids vif, quantités ingérées, variations.

\section{Introduction}

Le poids vif de la jument n'est pas un critère suffisant pour estimer les variations de poids et de composition du corps vide avec le niveau des apports alimentaires au cours du cycle gestation-lactation (Martin-Rosset \& Doreau, 1984 et Martin-Rosset, DOREAU \& ESPINASSE, 1986). Ainsi, au cours des derniers jours de la gestation chez la jument bien alimentée, le gain de poids vif peut être faible alors que le conceptus se développe car les quantités ingérées ont plutôt tendance à diminuer avec des régimes très grossiers, en raison de l'encombrement de plus en plus important du conceptus. En revanche, après mise bas, le poids vif de la jument s'accroît de 5 à 10 p. 100 (entre le $1^{\text {er }}$ et le $2^{\text {e }}$ mois de lactation) selon le niveau des apports alimentaires, alors que les quantités ingérées augmentent fortement pour faire face à l'accroissement important des dépenses de production laitière (Martin-Rosset \& Doreau, 1980 ; Henneke, Potter \& Kreider, 1981 ; Martin-Rosset, Doreau \& Espinasse, 1986).

Nous avons donc étudié une méthode indirecte qui permet d'estimer la variation de poids du contenu digestif lorsque la quantité ingérée varie sur de courtes périodes 
tandis que la masse corporelle reste à peu près constante. Nous avons mesuré chez la jument lourde la variation à court terme de son poids vif en fonction de l'augmentation ou de la diminution instantanée des quantités ingérées. Nous avons estimé que cette variation représentait en grande partie celle du contenu digestif.

\section{Matériel et méthodes}

\section{A. Animaux et régimes}

6 juments adultes et non gravides, de races Bretonne, Comtoise ou croisées ont été maintenues en stalles individuelles. Agées de $8,3 \pm 2,1$ ans, elles avaient un poids vif moyen de $789 \pm 64 \mathrm{~kg}$, un état corporel jugé satisfaisant et comparable au début de l'essai. Les juments ont eu chaque jour une heure d'exercice dans un paddock.

Le régime était composé de foin de pré et d'aliment concentré. La ration a été distribuée en 2 parties égales à 8 et $16 \mathrm{~h} 30$. Le fourrage et l'aliment concentré étaient distribués simultanément.

\section{B. Schéma expérimental}

Pendant 3 semaines ( $l^{r e}$ phase) les 6 juments ont reçu du foin de pré distribué ad libitum (10 p. 100 de refus) et $3,0 \mathrm{~kg}$ de concentré afin de stabiliser le poids de leur contenu digestif et les habituer à ce mode de conduite.

Les juments ont été séparées en deux lots de 3 animaux ( $2^{e}$ phase) sur la base des poids vifs et des quantités ingérées mesurées au cours des deux dernières semaines de la $1^{\text {re }}$ phase. Elles ont reçu du foin de pré distribué ad libitum et successivement 2 ou $4 \mathrm{~kg}$ d'aliment concentré. Nous avons mesuré pendant deux périodes successives de 7 jours, avec inversion entre 2 périodes des quantités de concentré distribuées: la capacité d'ingestion et les variations diurnes de poids vif.

Les 6 juments ont été ensuite conduites selon un dispositif en carré latin $\left(3^{e}\right.$ phase). Elles ont reçu pendant 6 périodes successives de 2 semaines, 2 ou $4 \mathrm{~kg}$ d'aliment concentré et, pour une quantité d'aliment concentré donnée du foin de pré distribué à différents niveaux représentant 95,80 et 60 p. 100 des quantités de foin ingérées pour une quantité d'aliment concentré correspondante ( 2 ou $4 \mathrm{~kg}$ ) et mesurée précédemment ( $2^{e}$ phase).

\section{Mesures}

\section{A. Pesées}

Au cours des $2^{\mathrm{e}}$ et $3^{\mathrm{e}}$ phases, les juments ont été pesées 5 jours consécutifs par semaine, respectivement 3 et 2 fois par jour : le matin à $8 \mathrm{~h}$ et le soir à $16 \mathrm{~h} 30$ avant chaque distribution et dans l'intervalle à $13 \mathrm{~h} 30\left(2^{e}\right.$ phase $)$ ou à $8 \mathrm{~h}$ et $13 \mathrm{~h} 30$ ( $3^{e}$ phase). 


\section{B. Composition chimique des aliments. Quantités ingérées et d'eau bue}

La teneur en matière sèche des aliments a été déterminée à l'étuve à $80^{\circ} \mathrm{C}$ et la composition chimique des aliments mesurée sur un échantillon moyen représentatif de l'ensemble de l'expérience. Le foin et l'aliment concentré présentaient respectivement des teneurs en cellulose brute de 33,2 et 5,1 p. 100 et en matières azotées totales de 8,8 et 15,8 p. 100 .

Les quantités de foin et d'aliment concentré offertes et refusées ont été pesées chaque jour avant la distribution du matin et au cours des $2^{\mathrm{e}}$ et $3^{\mathrm{e}}$ phases entre chacune des pesées journalières du poids vif. Nous avons estimé que la variation provoquée de matière sèche ingérée au cours d'un changement de niveau d'alimentation est égale à la différence entre les quantités ingérées moyennes des jours $\mathrm{J}-2$ et $\mathrm{J}-1$ d'une part, J0 et $\mathbf{J}+1$ d'autre part.

Nous avons mesuré, à l'aide d'un compteur volumétrique individuel, la quantité journalière d'eau bue par les juments, de même que la quantité d'eau bue entre chacune des différentes pesées journalières $\left(2^{\mathrm{e}}\right.$ et $3^{\mathrm{e}}$ phases).

\section{Analyse statistique des résultats}

Le dispositif en carré latin adopté au cours de la $3^{\mathrm{e}}$ phase ayant permis d'éliminer l'effet période, la variation de poids vif des juments ( $\mathrm{Y}$ exprimé en $\mathrm{kg}$ ) a été analysée, tous animaux confondus, en fonction des 2 variables étudiées : quantités de matière sèche ingérée de foin $\left(X_{1}\right.$ en $\left.\mathrm{kg}\right)$ et d'aliment concentré $\left(X_{2}\right.$ en $\left.\mathrm{kg}\right)$ par régression double.

\section{Résultats et discussion}

\section{A. Répétabilité entre jours de pesées en période d'alimentation stable}

Le poids vif des juments, mesuré à la même heure, est très répétable d'un jour à l'autre (tabl. 1). Les écarts types varient de 5 à $10 \mathrm{~kg}$ lorsque les juments reçoivent respectivement du foin à volonté ou en quantités limitées et 2 ou $4 \mathrm{~kg}$ de concentré. Le coefficient de variation est en moyenne de 1 p. 100, même lorsque les animaux sont nourris à volonté. Un tel résultat peut sans doute être expliqué par le nombre limité de défécations et de mictions quotidiennes, respectivement 1 toutes les 2 heures et 1 toutes les 4 heures (Blechschmidt, 1933 ; Hafez et al., 1969 ; TYler, 1972).

\section{B. Variation diurne du poids vif des juments recevant du foin à volonté et 2 ou $4 \mathrm{~kg}$ de concentré ( $2^{e}$ phase)}

Les quantités de matière sèche totale ingérée (MST) ont été très voisines : 19,3 et $19,6 \mathrm{~kg}$ bien que l'aliment concentré ait respectivement représenté 9,0 et 17,7 p. 100 de la MST. Les quantités d'eau bues ont été également très proches et en moyenne de 2,9 I par kg MS (tabl. 2). 
TABleau 1

Répétabilité entre jours des pesées en périodes d'alimentation stable.

Repeatability between days of weighing during constant feeding period.

\begin{tabular}{|c|c|c|c|c|c|c|}
\hline \multirow[b]{3}{*}{$\begin{array}{l}\text { Horaires de pesée } \\
\text { Time of weighing }\end{array}$} & \multicolumn{6}{|c|}{$\begin{array}{c}\left.\text { Foin ad libitum ( } 2^{\mathrm{c}} \text { phase }\right) \\
\text { Hay ad libitum }\end{array}$} \\
\hline & \multicolumn{3}{|c|}{$\begin{array}{r}+2 \mathrm{~kg} \text { concentré } \\
\text { concentrate }\end{array}$} & \multicolumn{3}{|c|}{$\begin{array}{r}+4 \mathrm{~kg} \text { concentré } \\
\text { concentrate }\end{array}$} \\
\hline & $8 \mathrm{~h}$ & 13 h 30 & $16 \mathrm{~h} 30$ & $8 \mathrm{~h}$ & 13 h 30 & $16 \mathrm{~h} 30$ \\
\hline $\begin{array}{l}\text { Quantités ingérées de foin ( } \mathrm{kg} \mathrm{MS} \text { ) } \\
\text { Hay intake ( } \mathrm{kg} \mathrm{DM})\end{array}$ & \multicolumn{3}{|c|}{$17,55 \pm 0,75$} & \multicolumn{3}{|c|}{$16,10 \pm 0,84$} \\
\hline $\begin{array}{l}\text { Poids moyen des juments }(\mathrm{n}=30) \\
\text { Livebody weight of the mares }\end{array}$ & 809,7 & 814,7 & 818,6 & 814,1 & 819,5 & 820,4 \\
\hline $\begin{array}{l}\text { Moyenne des écarts-types } \\
\text { des pesées journalières } \\
\text { des } 6 \text { juments }(\mathrm{kg}) \ldots \ldots \\
S D \text { of daily weights of the } \\
6 \text { mares }(\mathrm{kg})\end{array}$ & 4,7 & 5,4 & 4,6 & 5,1 & 6,1 & 4,9 \\
\hline
\end{tabular}

\begin{tabular}{|c|c|c|c|c|}
\hline \multirow[b]{2}{*}{$\begin{array}{l}\text { Alimentation } \\
\text { Feeding }\end{array}$} & \multicolumn{4}{|c|}{$\begin{array}{c}\text { Foin limité }\left(3^{\mathrm{e}} \text { phase }\right) \\
\text { Hay restricted }\end{array}$} \\
\hline & \multicolumn{2}{|c|}{$\begin{array}{c}\text { à } 60 \% \text { de l'ad libitum } \\
60 \% \text { of ad libitum } \\
+2 \text { ou } 4 \mathrm{~kg} \text { concentré } \\
\text { of concentrate }\end{array}$} & \multicolumn{2}{|c|}{$\begin{array}{l}\text { à } 95 \% \text { de l'ad libitum } \\
95 \% \text { of ad libitum } \\
+2 \text { ou } 4 \mathrm{~kg} \text { concentré } \\
\text { of concentrate }\end{array}$} \\
\hline $\begin{array}{l}\text { Horaires de pesées } \ldots \ldots \ldots \\
\text { Time of weighing }\end{array}$ & $8 \mathrm{~h}$ & 13 h 30 & $8 \mathrm{~h}$ & 13 h 30 \\
\hline $\begin{array}{l}\text { Poids moyen des juments }(n=30) \\
\text { Livebody weight of the mares }\end{array}$ & 806,8 & 817,7 & 817,1 & 841,3 \\
\hline $\begin{array}{l}\text { Moyenne des écarts-types } \\
\text { des pesées journalières } \\
\text { des } 6 \text { juments }(\mathrm{kg}) . . . \\
S D \text { of daily weights of the } \\
6 \text { mares }(\mathrm{kg})\end{array}$ & 8,4 & 6,7 & 9.6 & 8,0 \\
\hline
\end{tabular}




\section{Tableau 2}

Quantités de matière sèche et d'eau ingérées. Variations diurnes du poids vif chez des juments recevant du foin à volonté et $2 \mathrm{~kg}$ ou $4 \mathrm{~kg}$ d'aliment concentré ( $2^{e}$ phase).

Dry matter and water intake. Diurnal variations of livebody weight of mares fed forage ad libitum and 2 or $4 \mathrm{~kg}$ of concentrate $\left(2^{\text {nd }}\right.$ phase).

Quantité d'aliment concentré

Level of concentrate

Quantités ingérées :

Dry matter intake :

- Totales : kg MS/jour/animal total : $\mathrm{kg} \mathrm{DM/day/animal}$

- ${\text { g MS } / \mathbf{k g}^{0,75}}$

g DM/ $\mathrm{kg}^{0.75}$

- Foin kg MS/jour/animal Hay kg DM/day/animal

Eau bue :

Water intake :

- 1/jour/animal 1/day/animal

- $1 / \mathrm{kg} \mathrm{MS}$ $1 / \mathrm{kg} D M$

Variations diurnes du poids vif $(\mathrm{kg})(\mathbf{n}=30)$ :

Diurnal variations in livebody weight $(\mathrm{kg})$ :

- PV à 8 heures $L B W$ at $08.00 \mathrm{~h}$

- $\triangle \mathrm{PV} 8$ h 00-13 h 30 $\triangle L B W 08.00-13.30 h$

- $\Delta$ PV 13 h 30-16 h 30 $\Delta L B W 13.30-16.30 h$
$4 \mathrm{~kg}$

$19,58 \pm 2,35$

128

$16,10 \pm 2,35$

$17,55 \pm 2,57$

$16,10 \pm 2,35$

$55,10 \pm 17,90$

$55,50 \pm 18,80$

$2,85 \pm 1,08$

$2,83 \pm 1,19$

814,7

$+5,4 \pm 5,0$

$+0,8 \pm 4,7$

MS = Matière sèche $-D M=$ Dry Matter.

$\mathrm{PV}=$ Poids vif $-L B W=$ Livebody Weight.

$\Delta=$ Variation - Variation.

Les poids vifs mesurés 5 et 8 heures après le repas du matin sont toujours supérieurs à ceux observés avant, mais les différences restent inférieures à 1 p. $100 \mathrm{du}$ poids vif (tabl. 2). Elles sont plus limitées que celles observées chez la vache tarie nourrie à volonté avec des rations hivernales : 2 à 3 p. 100 du poids vif (PAQUAY, DE BAERE \& Lousse, 1971). Les différences mesurées (tous niveaux d'apport de concentré confondus) pourraient être dues aussi bien à la quantité d'eau bue : 29 p. 100 entre 8 et $13 \mathrm{~h} 30$ et 14 p. 100 entre 13 h 30 et 16 h 30 de l'abreuvement total, qu'à la quantité de matière sèche ingérée au cours des mêmes intervalles, respectivement : 29 et $10 \mathrm{p}$. 100 de la MST consommée. Sufit, Houpt \& SweEting (1985) ont en effet montré que l'essentiel de l'abreuvement est lié aux périodes d'ingestion. 
C. Variation de poids vif corrélative à une variation instantanée des quantités ingérées

Deux des 36 données prévues n'ont pu être obtenues avec la même jument recevant 2 ou $4 \mathrm{~kg}$ de concentré et 80 p. 100 de foin.

Les juments ont bien consommé 60 et 80 p. 100 des quantités ingérées ad libitum mais seulement 88 p. 100 au lieu de 95 p. 100 prévus (tabl. 3). La quantité d'eau bue a varié de 3 à $41 / \mathrm{kg}$ MS (tabl. 3) : elle est très comparable à celle mesurée par FonNESBECK (1968). Les quantités ingérées et d'eau bue entre les pesées de $8 \mathrm{~h}$ et 13 h 30 ont été d'autant plus élevées que les animaux étaient restreints (tabl. 3).

\section{TABLEAU 3}

Quantités ingérées et d'eau bue. Variations de poids vif chez des juments restreintes.

Dry matter and water intake. Variations of livebody weight in restricted mares.

\begin{tabular}{|c|c|c|c|c|}
\hline \multirow[b]{2}{*}{$\begin{array}{l}\text { Niveau de restriction théorique (\%) } \\
\text { Theoretical level of restriction }\end{array}$} & \multicolumn{3}{|c|}{$3^{\mathrm{e}}$ Phase } & \multirow{2}{*}{$\begin{array}{l}2^{\mathrm{e}} \text { Phase } \\
\text { ad libitum }\end{array}$} \\
\hline & 60 & 80 & 95 & \\
\hline $\begin{array}{l}\text { Niveau de restriction effectif }(\%) \ldots \ldots \\
\text { Applied level of restriction }\end{array}$ & 59,2 & 78,2 & 88,0 & 100 \\
\hline $\begin{array}{l}\text { Quantités ingérées de foin (kg MS) } \ldots \\
\text { Hay intake }(k g D M)\end{array}$ & 10,0 & 13,2 & 14,9 & 16,9 \\
\hline $\begin{array}{l}\text { Quantités d'eau bue : } \\
\text { Water intake: }\end{array}$ & & & & \\
\hline$\underset{l / d / \text { animal }}{1 / j / \text { animal }} \cdots \cdots \cdots \cdots \cdots \cdots$ & 31,0 & 51,7 & 50,4 & 55,3 \\
\hline $\begin{array}{l}\text { Variations entre } 8 \text { et } 13 \mathrm{~h} 30: \\
\text { Variations between } 08.00-13.30 \mathrm{~h} \text { : }\end{array}$ & & & & \\
\hline $\begin{array}{l}\text { - de la matière sèche totale ingérée } \ldots \\
\text { of total dry matter intake }\end{array}$ & 21 & 16 & 15 & 29 \\
\hline $\begin{array}{l}\text { - de l'abreuvement }(\%) \ldots \ldots \ldots \ldots \\
\text { of water intake }\end{array}$ & 43 & 36 & 33 & 29 \\
\hline $\begin{array}{l}\text { - du poids vif } \ldots \ldots \ldots \ldots \ldots \\
\text { of livebody weight }\end{array}$ & $+10,9$ & $+7,5$ & $+4,2$ & $+\quad 5,2$ \\
\hline
\end{tabular}

Le poids vif mesuré à $8 \mathrm{~h}$ est toujours inférieur à celui observé à $13 \mathrm{~h} 30$. L'écart est toujours inférieur à 2 p. 100 du poids vif des juments, mais plus élevé avec 60 p. 100 de restriction qu'à 95 p. 100.

Le poids vif de la jument ne varie significativement qu'après un délai de 24 heures (J0) suivant un changement des quantités de foin ou d'aliment concentré distribuées. Ce délai correspond en grande partie au temps de séjour des aliments dans le gros intestin. Il est rapidement stabilisé 48 heures $(\mathrm{J}+1)$ à 72 heures $(\mathrm{J}+2)$ après car le transit 
total des aliments dans le tube digestif du cheval est seulement de 28 à 48 heures (Olsson et al., 1949 ; Wolter et al., 1974 ; UdEN et al., 1982) et il ne varie pas significativement avec le niveau d'alimentation (Miraglia, Poncet \& Martin-Rosset, données non publiées). La variation de poids vif $(\mathrm{Y})$ est bien reliée à la variation de quantité de matière sèche ingérée de fourrage $\left(X_{1}\right)$ et d'aliment concentré $\left(X_{2}\right)$ :

$$
\mathrm{Y}=1,636+3,48 \mathrm{X}_{1}+3,45 \mathrm{X}_{2} ; \mathrm{n}=29 ; \mathrm{r}=0,88 * *
$$

Le poids vif de la jument varie de $3,48 \pm 0,40 \mathrm{~kg}$ ou de $3,45 \pm 0,95 \mathrm{~kg}$ lorsque les quantités de matière sèche ingérée de fourrage ou d'aliment concentré varient respectivement de $1 \mathrm{~kg}$. La précision de la variation est plus élevée dans le cas des fourrages $(\mathrm{CV}=11,5$ p. 100) que dans celui de l'aliment concentré $(\mathrm{CV}=27,5 \mathrm{p} .100)$. Cette valeur est légèrement plus faible que celle mesurée chez la vache laitière en lactation : 3,6 à 4,5 kg (Piton, 1975 ; Verite \& Journet, 1975).

\section{Conclusion}

La variation à court terme des quantités ingérées se traduit par une variation importante du poids vif, qui peut être interprétée par une variation du contenu digestif sans que celle-ci ait été ici directement mesurée. Cette relation est très utile pour interpréter la stabilisation du poids vif de la jument, observée en fin de gestation et concomitante à la diminution des quantités ingérées mesurées : 10 p. 100 au cours des 70 derniers jours (Martin-Rosset \& Doreau, 1980). Elle peut expliquer également en partie l'augmentation importante de poids vif observé en début de lactation : 5 à $10 \mathrm{p}$. 100 du poids vif mesuré à la mise bas (Martin-Rosset \& Doreau, 1980 ; Banach \& Evans, 1981 ; Henneke, Potter \& Kreider, 1981 ; Zimmerman, 1981 ; Martin-Rosset \& DOREAU, 1984), suite à l'augmentation considérable des quantités ingérées : 40 p. 100 au cours des huit $1^{\text {res }}$ semaines (Martin-Rosset \& Doreau, 1980 ; Boulot \& Doreau, 1986). Mais dès la $3^{\mathrm{e}}$ ou $4^{\mathrm{e}}$ semaine, la jument paraît être en bilan énergétique positif lorsqu'elle est alimentée à volonté (Doreau, Boulot \& Martin-Rosset, en préparation) et pourrait constituer des réserves corporelles comme semble l'indiquer la décroissance de la teneur plasmatique en AGNE mesurée au cours des 2 premiers mois de lactation (Boulot \& Doreau, 1986).

Dans ces conditions, il faut, pour interpréter la totalité de la variation de poids vif observée en début de lactation, utiliser à la fois la relation proposée pour estimer la variation de poids du contenu digestif et une relation d'estimation indirecte des réserves corporelles. Cette dernière relation reste à établir.

Reçu en décembre 1985.

Accepté en septembre 1986. 


\section{Summary}

\section{Simultaneous variations of livebody weight and feed intake in dry mares}

Short-term variations in livebody weight following induced variations in feed intake were measured in 6 heavy dry mares fed meadow hay supplemented with 2 or $4 \mathrm{~kg}$ concentrate. Amounts of hay offered represented 95,80 , and 60 p. 100 of the voluntary feed intake according to a latin square design. Short-term variations in livebody weight were assumed to provide reliable estimations of variations in the digestive contents.

Livebody weight variations $(\mathrm{Y})$ were fairly well related in forage $\left(\mathrm{X}_{1}\right)$ or concentrate $\left(\mathrm{X}_{2}\right)$ dry matter (DM) intake. When variations in forage or concentrate DM intake were $1 \mathrm{~kg}$, those of livebody weight of the mare were $3.48 \pm 1.40$ and $3.45 \pm 0.95 \mathrm{~kg}$, respectively.

Key words : Horse, mare, livebody weight, feed intake, variations.

\section{Références bibliographiques}

Banach M.A., Evans J.W., 1981. Effects of inadequate energy during gestation and lactation on the oestrous cycle and conception rates of mares and on their foal weights. Proc. 7th Equine Nutr. Physiol. Symp., 97-100.

BlechschmidT H., 1933. Experimentaluntersuchungen und Ermittlungen über die Ernärhung des Pferdes auf der Weide. Landw. $J b .77,463-560$.

Boulot S., Doreau M., 1986. Effets de l'état physiologique et du régime alimentaire sur quelques paramètres sanguins chez la jument. $37^{\mathcal{e}}$ Réunion F.E.Z. Budapest 1-4 septembre, Commission Chevaline, Session 1, poster.

Boulot S., Doreau M., 1986. Effect of voluntary intake in the mare during late pregnancy and early lactation: effect of diet. 37th E.A.A.P. meeting Budapest, Commission on Horse Production, Session 1, $13 \mathrm{p}$.

FonNESBECK P.V., 1968. Consumption and excretion of water by horses receiving all hay and haygrain diets. J. Anim. Sci., 27, 1350-1356.

Hafez E.S.E., Williams M., WIERzbowsKI S., 1969. The behaviour of Horses. In Hafez E.S.E. Ed. Behaviour of domestic animals, Baillere Tindall Cox. London, p. 360-395.

Henneke D.R., Potter G.D., Kreider J.L., 1981. Rebreeding efficiency in mares fed different levels of energy during late gestation. Proc. 7th Equine Nutr. Physiol. Symp., 111-115.

Martin-Rosset W., Doreau M., 1980. Effect of variation in level of feeding of heavy mares during late pregnancy. 31st E.A.A.P. meeting Munich. Commission on Horse Production, Session 4, 7 p.

Martin-Rosset W., Doreau M., 1984. Besoins et Alimentation de la jument, 355-370. In R. Jarrige et W. Martin-Rosset. Ed : Le Cheval : Reproduction, Sélection, Alimentation, Exploitation. I.N.R.A. Publications, Route de Saint-Cyr, 78000 Versailles, p. 689.

Martin-Rosset W., Doreau M., Espinasse R., 1986. Alimentation de la jument lourde allaitante : Evolution du poids vif et croissance des poulains. Ann. Zootech., 35, 21-36.

Olsson N., Kinlen G. et Cagell W., 1949. Digestibility experiments on horses and evacuation experiments to investigate the time required for the food to pass through the horse's digestive tract., Lantbrukshogsk. Husdjursforsoks. Medd., 36, 1-51.

Paquay R., de Baere R., Lousse A., 1971. Des variations du poids vif chez la bête bovine. Rev. Agricult., 4, 419-429. 
Piton J., 1975. Intérêt et importance de la mobilisation des réserves corporelles des vaches laitières au début de la lactation. Analyse de quelques facteurs de variation. Thèse. Université des Sciences et Techniques du Languedoc. Montpellier.

Sufit E., Houpt K.A., Sweeting M., 1985. Physiological stimuli of thirst and drinking patterns in ponies. Eq. Vet. J., 17, 12-16.

TYler S.J., 1972. The behaviour and social organization of the new forest ponies. Anim. Behav. Monogr., 5, 87-196.

Uden P., Rounsaville T.R., Wiggans G.R., Van Soest P.J., 1982. The measurement of liquid and solid digesta retention in ruminants, equines and rabbits given timothy (Phleum pratense) hay. Br. J. Nutr., 48, 329-339.

Verite R., Journet M., 1975. Alimentation des vaches laitières avec de l'ensilage de maìs : influence de la nature de l'ensilage, de la suralimentation énergétique et de la nature de la complémentation azotée. Ann. Zootech., 24, 95-107.

Wolter R., Durix A., Letourneau J.C., 1974. Influence du mode de présentation du fourrage sur la vitesse du transit digestif chez le poney. Ann. Zootech., 23, 293-300.

Zimmerman R.A., 1981. Energy needs of lactating mares. Proc. 7th Equine Nutr. Physiol. Symp., 127-135. 